\title{
Path Integral Monte Carlo Simulations of Warm Dense Sodium
}

\author{
Shuai Zhanga,*, Kevin P. Driver ${ }^{\mathrm{a}}$, François Soubiran ${ }^{\mathrm{a}}$, Burkhard Militzer ${ }^{\mathrm{a}, \mathrm{b}, *}$ \\ ${ }^{a}$ Department of Earth and Planetary Science, University of California, Berkeley, California 94720, USA \\ ${ }^{b}$ Department of Astronomy, University of California, Berkeley, California 94720, USA
}

\begin{abstract}
High energy density physics and astrophysics require reliable methods for determining the equation of state of warm dense matter. At high temperatures (above $10^{6} \mathrm{~K}$ or $100 \mathrm{eV}$ ), path integral Monte Carlo (PIMC) as a first-principles method is a useful option because of its accuracy and efficiency. Previous developments in PIMC implemented free-particle nodes to study plasmas comprised of heavy $(Z \leq 10)$ elements, and constructed equations of state in tandem with those from molecular dynamics (MD) simulations based on density functional theory (DFT), whose applicability is limited to low temperatures (up to $\sim 10^{6}$ K). Recent PIMC method developments employed a localized, Hartree-Fock nodal surface, allowing for a better description of bound states in warm dense silicon. In this work, we use the localized nodal scheme to study warm dense sodium. We demonstrate that PIMC and DFT-MD produce a coherent equation of state, and discuss the electronic structure of the plasma.
\end{abstract}

Keywords: Sodium, Equation of state, Path integral Monte Carlo, Density functional theory

\section{Introduction}

High energy density physics and astrophysics require reliable equations of state (EOS) 3 for warm dense matter. In the limit of high temperature (above $10^{3} \mathrm{eV}$ ), matters transition 4 into weakly-interacting plasmas because of strong thermal ionization. In these occasions, 5 the uniform electron gas model (ideal Fermi gas theory) and the Debye-Hückel theory are 6 good approximations. At low to intermediate temperatures (below $100 \mathrm{eV}$ ), molecular dy7 namics (MD) simulations based on Kohn-Sham density functional theory (DFT) are suitable 8 options, because of their accuracy in electronic structure calculations. However, standard 9 DFT-MD is inefficient at higher temperatures, due to an increasingly large number of ex- cited states, which make the calculation intractable. The path integral Monte Carlo (PIMC) 1 method provides a framework to bridge the gap between DFT-MD and the high temperature

\footnotetext{
${ }^{*}$ Corresponding authors.

Email addresses: shuai.zhang01@berkeley.edu (Shuai Zhang), militzer@berkeley.edu (Burkhard Militzer)

Preprint submitted to High Energy Density Physics

August 30, 2016
} 
limit. Using free-particle (FP) nodes in PIMC, we produced EOS across the entire WDM regime for several heavy elements $(2<Z \leq 10)$ and compounds $\left(\mathrm{H}_{2} \mathrm{O}\right)[1,2,4,4,4,5]$.

Several attempts have been made to go beyond FP nodes in PIMC simulations [6, 7]. In recent work, we introduced localized nodal surfaces in PIMC simulations to treat bound electron states, which allows for simulations of third-period elements in the WDM regime, such as silicon [8]. Our results for an isolated silicon atom showed that nodes derived from Hartree-Fock (HF) orbitals yield highly accurate predictions for the pressure and the internal energy. The combined results using this PIMC method and DFT-MD provide a coherent EOS for dense silicon plasmas over a wide density-temperature grid $\left(2.3-18.6 \mathrm{~g} / \mathrm{cm}^{3}, 5 \times 10^{5}\right.$ $\left.1.3 \times 10^{8} \mathrm{~K}\right)$.

In addition to high temperatures that limit its efficiency and applicability, low densities also raise challenges to DFT-MD. As the density of a system decreases, DFT-MD requires an increasingly larger number of plane waves to describe the states and is more difficult to converge. On the other hand, PIMC operates in the real space, therefore the computational cost of a single Monte Carlo move does not depend on density.

In this study, we apply PIMC to study sodium. $\mathrm{Na}$ is an element that has been of wide interest [9] since it is traditionally viewed as a "simple metal" yet adopts a series of non-close-packed phases under high pressure [10], associated with changes in optical and electronic properties [11, 12] and melting behavior [13, 14]. It was also found that $\mathrm{Na}$ turns insulating at $200 \mathrm{GPa}$ and re-metalizes at $15.5 \mathrm{TPa}$ [15, 16, 17.

The results from the present study are instructive to exhaustive EOS calculations at other densitites and shock-compression predictions, which can guide future work on $\mathrm{Na}$ at high-pressure, temperature conditions, characteristic of deep interiors of giant planets and stars, planetary impacts, or thermonuclear fusion, and help build more accurate models for astrophysical applications.

\section{Calculation methods}

Our simulations use an 8-atom cell, except for some low-temperature cases of DFT-MD, for which a large cell with 54 atoms is computationally affordable. We present results for density equaling $1.933 \mathrm{~g} / \mathrm{cm}^{3}, 2$ times the ambient density $\left(\rho_{\text {ambient }}\right)$ of solid sodium at room temperature and in the body-centered cubic phase [18. We consider a temperature range of $10^{3}-1.29 \times 10^{8} \mathrm{~K}$, relevant to conditions at stellar interiors. This is computationally challenging for both PIMC and DFT-MD.

Our PIMC simulations are based on the CUPID code [19]. The Coulomb interaction is introduced through pair density matrices [20]. The nodes are enforced at intervals of 1/8192 Hartrees (Ha), which means between 2560 and 20 time slices are needed for simulations in the temperature range of 1-129 million $\mathrm{K}$. It is sufficient to evaluate the pair action only at intervals of $1 / 1024 \mathrm{Ha}$ [7].

We employ the fixed-node approximation [21] to treat the fermion sign problem. The nodal surface is generated by introducing HF orbitals into the trial density matrix [8]. The HF orbitals are computed with the GAMESS code [22] and expanded in a localized basis set $(6-31+G)$. In order to overcome a rapidly decaying acceptance ratio of the ion moves 
that is caused by introducing the atomic orbitals, we enforce multi-particle moves that were introduced in 8 to displace each nucleus and its nearby electrons together as a group, which leads to efficient ion moves.

In DFT-MD simulations, we make use of the local density approximation (LDA) [23, 24] for the exchange-correlation functional. We choose a projected augmented wave pseudopotential [25, 26] that treats $1 s^{2}$ as core electrons and has a small core radius of $1.45 \mathrm{Bohr}$, plane wave basis with $4000 \mathrm{eV}$ cutoff, $\Gamma$-point for sampling the Brilliouin zone, and a time step of $0.2 \mathrm{fs}$. The typical length of MD simulations exceed $0.4 \mathrm{ps}$.

In order to correct the difference in energy references, we compare the energies of an isolated Na computed from an all-electron method (with the OPIUM code [27]) and a DFT pseudopotential in a box of $5 \times 5 \times 5 \mathrm{Bohr}^{3}$ (with VASP). We determine a shift of -161.3386 Ha/atom should be applied to all VASP internal energies.

\section{Results and discussion}

\subsection{Equation of state}

We calculate internal energies and pressures at a series of temperatures ranging from $10^{3}$ to $2 \times 10^{6} \mathrm{~K}$ using DFT-MD, and from $10^{6}$ to $1.29 \times 10^{8} \mathrm{~K}$ using PIMC. In order to verify the consistency of present PIMC results with existing EOS theories for warm dense matter, we compare the calculated internal energies and pressures with the Debye-Hückel model, which screens each electron in a spherical region to approximate the deviation from ideal plasmas, and the ideal Fermi gas model, which is exact when the system is fully ionized by temperature into a uniform electron gas.

The results are summarized in Table 1 and Fig. 1. Our calculations show that, both internal energies and pressures increase with temperature, because of the growing contribution of the kinetic energy and the thermal pressure. At low temperatures, the ideal Fermi gas model significantly overestimates the energy and the pressure, because of the neglection of bound-state energies and their contributions to the pressure. The curves are lowered down in the Debye-Hückel model, which introduces a screening length that better counts the deviation from ideal plasmas. However, because of its simplicity, the accuracy of the Debye-Hückel model is questionable at low temperatures when thermal ionization is weak.

At $2 \times 10^{6} \mathrm{~K}$, we find excellent agreement between PIMC and DFT-MD. The internal energy differs by less than $0.5 \mathrm{Ha} /$ atom, and the pressure agrees to within $2.7 \%$. At high temperatures of $T>2 \times 10^{7} \mathrm{~K}$, our PIMC results agree well with the Debye-Hückel model as well as the Fermi gas theory, because the temperatures are so high that the atoms are fully ionized. As $T$ decreases, the system gradually deviates from the ideal Fermi gas and becomes better described by the Debye-Hückel model, until $\sim 6 \times 10^{6} \mathrm{~K}$.

At 1 million K, the consistence between PIMC and DFT-MD is not as good but still remarkable - within $7.3 \mathrm{Ha}$ /atom in energy and $16.6 \%$ in pressure. We attribute this to the overlap of electronic orbitals at low temperatures, which degrades the quality of localized orbitals in the fixed-node approximation and reduces the sampling efficiency of PIMC. Below $2 \times 10^{6} \mathrm{~K}$, DFT-MD is capable in predicting the correct energies and pressures. 
Table 1: EOS table of sodium at $\rho=1.93328 \mathrm{~g} / \mathrm{cm}^{3}$ from DFT-MD (LDA) and PIMC simulations in this work.

\begin{tabular}{|c|c|c|c|c|c|}
\hline$\overline{\text { Method }}$ & $\begin{array}{c}T \\
{[\mathrm{~K}]}\end{array}$ & $\begin{array}{c}E \\
{[\text { Ha/atom }]}\end{array}$ & $\begin{array}{c}\sigma(E) \\
\text { [Ha/atom] }\end{array}$ & $\begin{array}{c}P \\
{[\mathrm{GPa}]}\end{array}$ & $\begin{array}{c}\sigma(P) \\
{[\mathrm{GPa}]}\end{array}$ \\
\hline $\operatorname{LDA}^{a}$ & 1000.00 & -161.373275 & 0.000456 & 14.1876 & 0.0541 \\
\hline $\operatorname{LDA}^{\frac{-a}{a}}$ & 10000.00 & -161.292271 & 0.001864 & 28.0283 & 0.2268 \\
\hline $\mathrm{LDA}^{b}$ & 50523.50 & -160.866617 & 0.001832 & 87.7294 & 0.5980 \\
\hline $\mathrm{LDA}^{\text {芴 }}$ & 67364.70 & -160.578654 & 0.001404 & 113.6573 & 0.6299 \\
\hline $\mathrm{LDA}^{\mathrm{b}}$ & 101047.00 & -159.710769 & 0.001477 & 185.9401 & 1.3009 \\
\hline $\mathrm{LDA}^{\mathrm{b}}$ & 126309.00 & -158.894894 & 0.001986 & 247.3363 & 1.4924 \\
\hline $\mathrm{LDA}^{\mathrm{b}}$ & 202095.00 & -155.904890 & 0.001458 & 477.1452 & 0.9449 \\
\hline $\mathrm{LDA}^{b}$ & 252619.00 & -153.559287 & 0.002389 & 659.3251 & 0.8812 \\
\hline $\mathrm{LDA}^{b}$ & 505239.00 & -139.389141 & 0.012724 & 1842.3810 & 2.9380 \\
\hline $\mathrm{LDA}^{\mathrm{b}}$ & 673652.60 & -128.866887 & 0.036004 & 2843.2556 & 3.3124 \\
\hline $\mathrm{LDA}^{\mathrm{b}}$ & 842065.80 & -118.079891 & 0.003260 & 3859.0165 & 1.0447 \\
\hline $\mathrm{LDA}^{\mathrm{b}}$ & 1010479.00 & -107.529075 & 0.009934 & 5021.0920 & 4.7770 \\
\hline $\mathrm{LDA}^{\mathrm{b}}$ & 2020958.00 & -49.310792 & 0.111457 & 12166.1570 & 11.0920 \\
\hline PIMC & 1010479 & -107.928000 & 1.466000 & 4186.7000 & 216.1000 \\
\hline PIMC & 2020958 & -42.076000 & 1.368000 & 11839.1000 & 201.4000 \\
\hline PIMC & 4041916 & 133.096000 & 1.199000 & 29481.7000 & 173.0000 \\
\hline PIMC & 8083831 & 420.284000 & 1.536000 & 64923.1000 & 225.7000 \\
\hline PIMC & 16167663 & 891.233000 & 2.071000 & 133306.5000 & 304.7000 \\
\hline PIMC & 32335325 & 1821.117000 & 2.779000 & 269840.0000 & 408.8000 \\
\hline PIMC & 64670651 & 3665.296000 & 3.288000 & 541096.5000 & 483.7000 \\
\hline PIMC & 129341301 & 7348.466000 & 3.249000 & 1083108.6000 & 478.2000 \\
\hline
\end{tabular}

${ }^{a} 54$-atom cell

${ }^{b} 8$-atom cell 

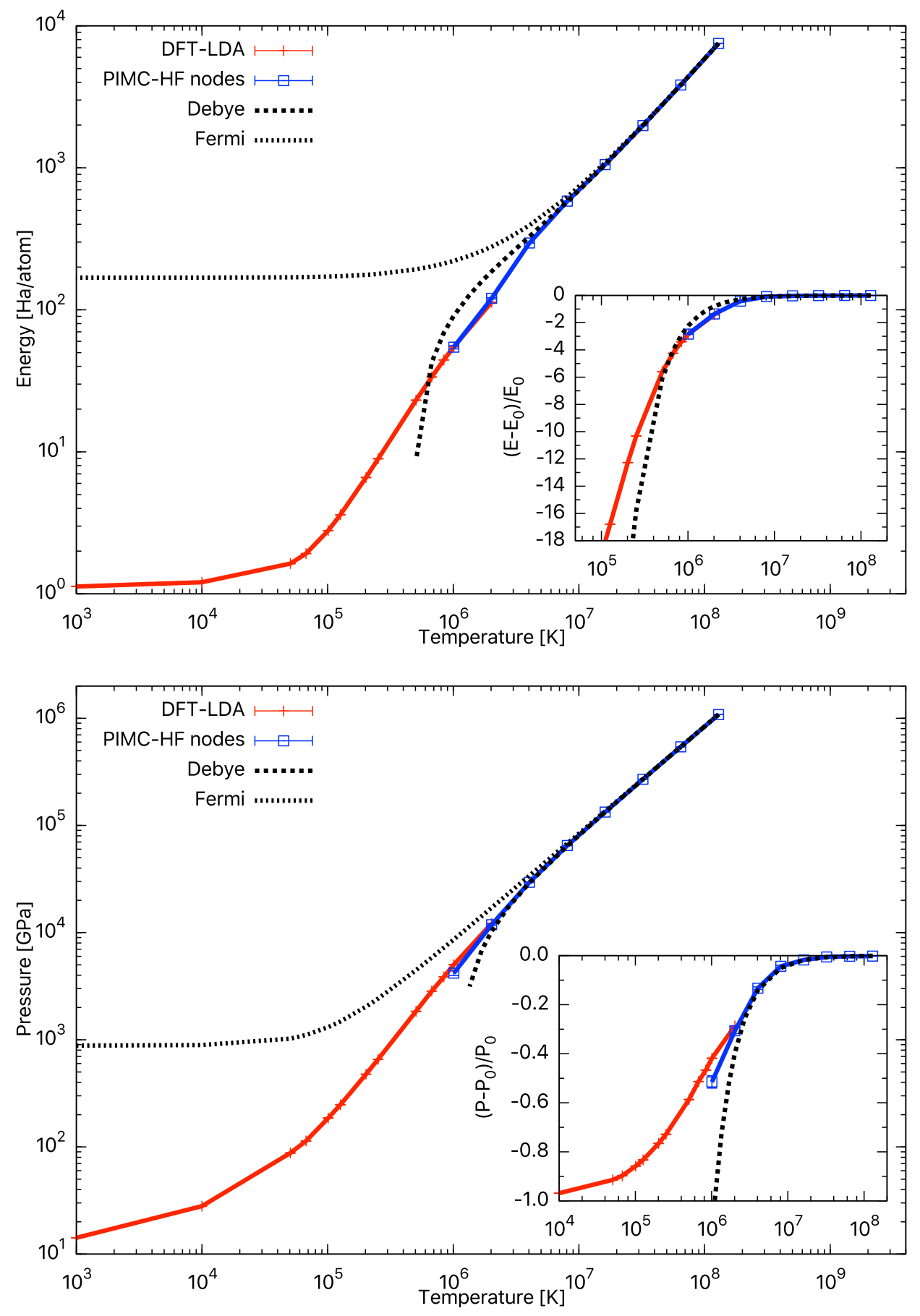

Figure 1: Energies and pressures of sodium along an isochore $\left(\rho=2 \rho_{\text {ambient }}\right)$ from first-principles (DFT and PIMC) computations, in comparison with that from the Debye-Hückel theory and the ideal Fermi electron gas model. Insets are the relative differences in comparison with the ideal Fermi electron gas model. In the upper panel, the energies have been made positive by shifting up by $162.5 \mathrm{Ha} /$ atom for logscale plotting. The agreements of PIMC with DFT at $2 \times 10^{6} \mathrm{~K}$, and with analytic models above $2 \times 10^{7}$ $\mathrm{K}$, are remarkable. 


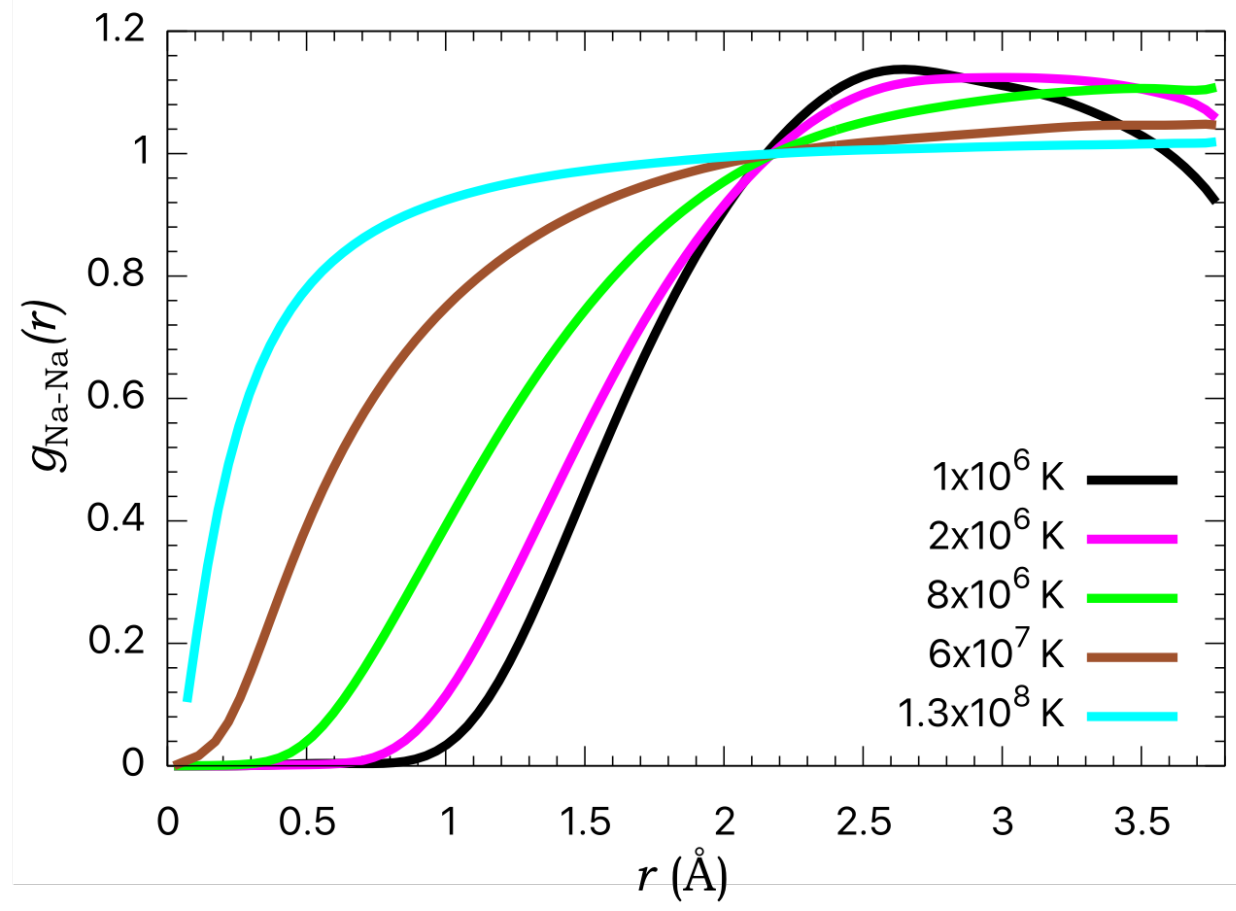

Figure 2: Evolution of the PIMC calculated nuclear pair correlation function with temperature.

The success in bridging analytical models in the high-temperature limit, and DFT-MD at $2 \times 10^{6} \mathrm{~K}$, validates the EOS data from present PIMC simulations with fixed-node approximation, and the use of a frozen core and a zero-temperature exchange-correlation functional in DFT-MD.

\subsection{Ionic and electronic plasma structure}

In Fig. 2, we compare the nuclear pair correlation functions $g_{\mathrm{Na}-\mathrm{Na}}(r)$ from PIMC simulations at elevated temperatures. As temperature increases, the kinetic energy surpasses the Coulomb repulsion, decreasing the threshhold in distance to find a neighboring atom. This reduces the correlation in the motion of the nuclei overall and flattens the peaks in $g_{\mathrm{Na}-\mathrm{Na}}(r)$. The gradual loss of the peak feature in $g_{\mathrm{Na}-\mathrm{Na}}(r)$ with increasing temperature is consistent with the expectation that the nuclei distribute more uniformly as electrons approach an ideal Fermi gas.

The temperature-driven ionization process, as used in the previous section for explaining the consistency in energy and pressures of PIMC with plasma models and DFT-MD, is illustrated in Fig. 3 via plots of the number of eletrons near the nucleus $N_{\mathrm{Na}-e}(r)$. Different stages of electron ionization can be observed by comparing $N_{\mathrm{Na}-e}(r)$ from PIMC with that of electronic states of a hydrogen-like sodium ion. The results show that $N_{\mathrm{Na}-e}(r)$ decreases with $T$, indicating the gradual thermal ionization. The good match between the calculated $N_{\mathrm{Na}-e}(r)$ profile at $2 \times 10^{6} \mathrm{~K}$ and that of $\mathrm{Na}^{9+}$ at $r=0-0.2 \mathrm{Bohr}$ indicates the 1 s electrons are barely excited at below $2 \times 10^{6} \mathrm{~K}$, validating the use of the pseupotential in DFTMD. Moreover, the increasing slope with $r$ and proximity of the $N_{\mathrm{Na}-e}(r)$ profiles at above 


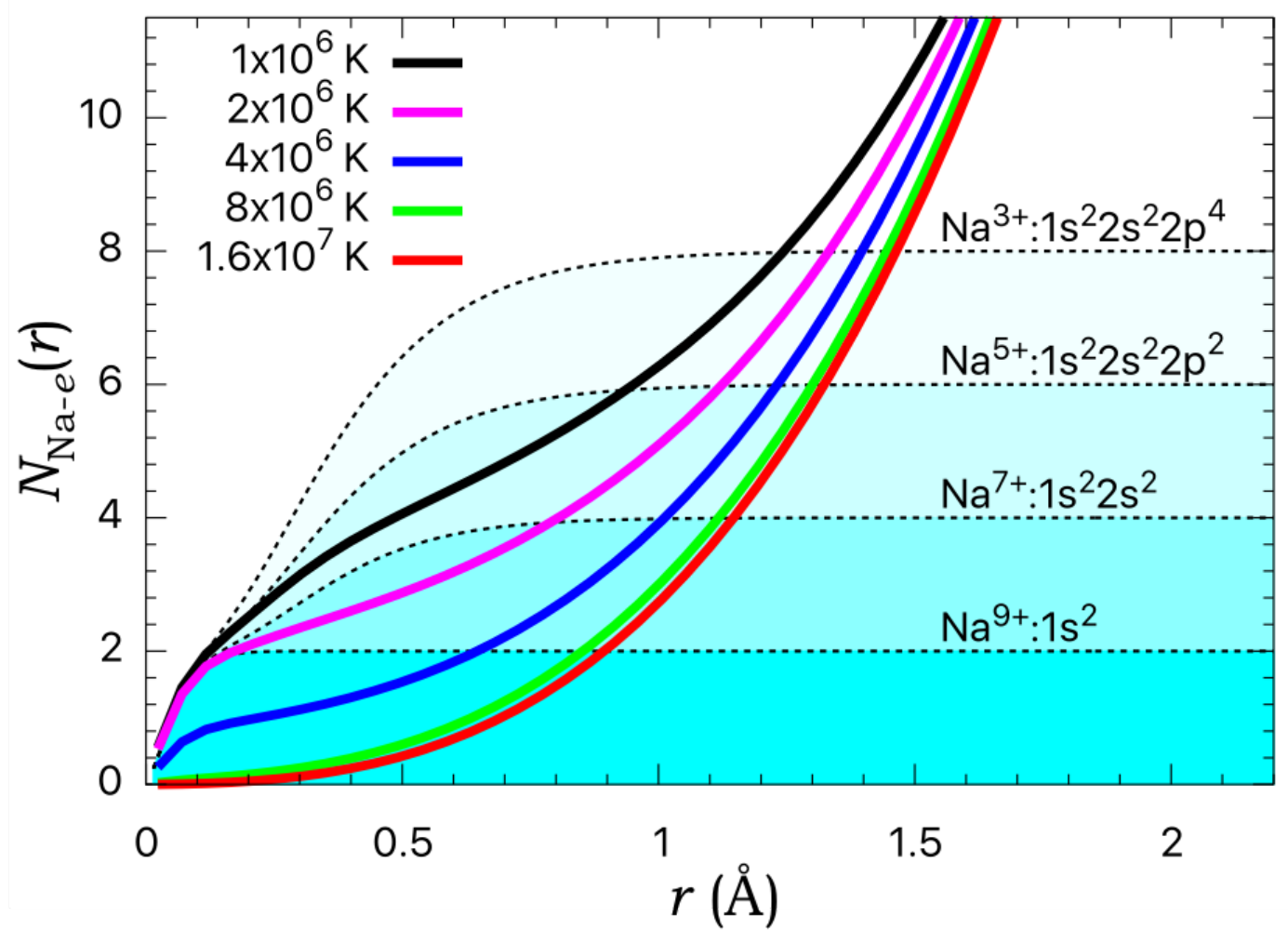

Figure 3: Number of electrons near each nucleus at $\rho=1.933 \mathrm{~g} / \mathrm{cm}^{3}$ and a series of temperatures. The profiles of four different electronic states of a hydrogen-like sodium ion are also shown for comparison.

$8 \times 10^{6} \mathrm{~K}$ show the electron distribution becomes stable over temperature. This implies a near-completion of the thermal ionization process and is consistent with the agreement in EOS between PIMC and plasma models at those temperatures discussed previously in Sec. 3.1.

In Fig. 4 we investigate the pair correlation function for electrons with opposite and parallel spins, and their evolution with temperature. The results show that, at $T \leq 4 \times 10^{6}$ $\mathrm{K}$, the maximum of $g_{e^{\uparrow}-e^{\downarrow}}(r)$ are at $r \rightarrow 0$, because multiple electrons occupy bound states of one nucleus. With increasing temperature, the magnitute is decreased as an increasing number of electrons are excited to higher-energy states and become unbound.

Electrons with parallel spins are regulated by Pauli repulsion. Therefore, $g_{e^{\uparrow}-e^{\uparrow}}(r)$ is brought down to 0 as $r \rightarrow 0$, giving rise to peaks in the correlation profiles at $r \approx 0.15 \AA$.

When $T>8 \times 10^{6} \mathrm{~K}$, the pair correlation functions of electrons with opposite and parallel spins are similar to each other at $r>0.12 \AA$, because the strong ionization and high kinetic effects surpass the Coulomb interaction, and the electrons resemble classical particles due to the short thermal de Broglie wavelength. The feature and difference $r<0.12 \AA$ are due to the complicated competition between ionization, kinetics, and Coulomb repulsion of all electrons, and the Pauli repulsion between electrons with parallel spins. 


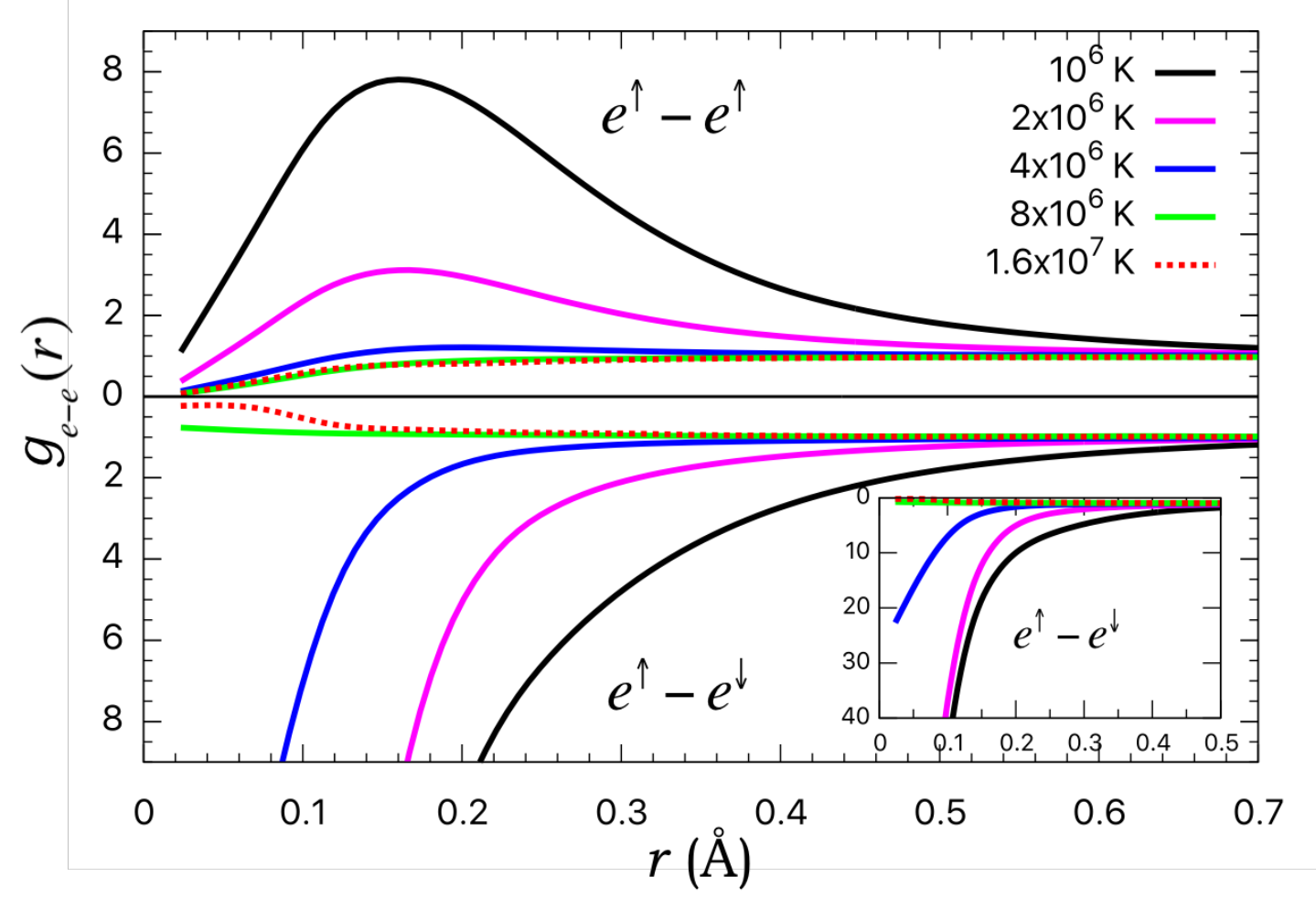

Figure 4: Pair correlation function of electrons at a series of temperatures.

\section{Conclusion}

We have shown that, our PIMC simulations with localized, HF nodes yield remarkable agreement with analytical plasma models at $T>6 \times 10^{6} \mathrm{~K}$, and with DFT-MD at $2 \times 10^{6}$ $\mathrm{K}$, in both internal energies and pressures. The results validate the EOS data from present PIMC simulations with fixed-node approximation, and the use of a frozen core and a zerotemperature exchange-correlation functional in DFT-MD.

Analysis of the thermal ionization process of sodium has shown that $1 s^{2}$ electrons become excited at $T>2 \times 10^{6} \mathrm{~K}$. Therefore DFT-MD with pseudopotentials freezing the $1 s$ electrons are inappropriate for studies at those high-temperature conditions, at $\rho=1.933 \mathrm{~g} / \mathrm{cm}^{3}$.

Our PIMC simulations have enabled us to study the ionic and electronic plasma structures of sodium at a series of temperatures, and we have been able to construct a coherent first-principles EOS table (Table 1) for warm dense sodium at $1.933 \mathrm{~g} / \mathrm{cm}^{3}$ and over a wide range of temperatures $\left(10^{3}-1.29 \times 10^{8} \mathrm{~K}\right)$.

Acknowledgments. The work has been supported by DOE (DE-SC0010517 and DESC0016248). Computational resources were provided by the Janus supercomputer, which is supported by the NSF (CNS-0821794), the University of Colorado, and the National Center for Atmospheric Research, and NERSC. 


\section{References}

[1] K. P. Driver, B. Militzer, All-Electron Path Integral Monte Carlo Simulations of Warm Dense Matter: Application to Water and Carbon Plasmas, Phys. Rev. Lett. 108 (2012) 115502.

[2] K. P. Driver, B. Militzer, First-Principles Equation of State Calculations of Warm Dense Nitrogen, Phys. Rev. B 93 (2016) 064101.

[3] K. P. Driver, F. Soubiran, S. Zhang, B. Militzer, First-principles equation of state and electronic properties of warm dense oxygen, J. Chem. Phys. 143 (2015) 164507.

[4] K. P. Driver, B. Militzer, First-principles simulations and shock hugoniot calculations of warm dense neon, Phys. Rev. B 91 (2015) 045103.

[5] K. P. Driver, F. Soubiran, S. Zhang, B. Militzer, Comparison of Low-Z Plasmas in Path Integral Monte Carlo Simulations, within this Proceeding.

[6] S. A. Khairallah, J. Shumway, E. W. Draeger, Path Integral Calculations of the Hydrogen Hugoniot Using Augmented Nodes arXiv:1108.1711.

[7] B. Militzer, D. M. Ceperley, Path integral monte carlo calculation of the deuterium hugoniot, Phys. Rev. Lett. 85 (2000) 1890-1893.

[8] B. Militzer, K. P. Driver, Development of path integral monte carlo simulations with localized nodal surfaces for second-row elements, Phys. Rev. Lett. 115 (2015) 176403.

[9] J. L. Dye, The alkali metals: 200 years of surprises, Phil. Trans. R. Soc. A 373 (2015) 20140174.

[10] E. Gregoryanz, L. F. Lundegaard, M. I. McMahon, C. Guillaume, R. J. Nelmes, M. Mezouar, Structural diversity of sodium, Science 320 (2008) 1054-1057.

[11] A. Lazicki, A. F. Goncharov, V. V. Struzhkin, R. E. Cohen, Z. Liu, E. Gregoryanz, C. Guillaume, H.-K. Mao, R. J. Hemley, Anomalous optical and electronic properties of dense sodium, Proc. Natl. Acad. Sci. USA 106 (2009) 6525-6528.

[12] J. Ibañez Azpiroz, B. Rousseau, A. Eiguren, A. Bergara, Ab initio analysis of plasmon dispersion in sodium under pressure, Phys. Rev. B 89 (2014) 085102.

[13] M. Marqués, M. Santoro, C. L. Guillaume, F. a. Gorelli, J. Contreras-García, R. T. Howie, A. F. Goncharov, E. Gregoryanz, Optical and electronic properties of dense sodium, Phys. Rev. B 83 (2011) 184106.

[14] H. Eshet, R. Z. Khaliullin, T. D. Kühne, J. Behler, M. Parrinello, Microscopic origins of the anomalous melting behavior of sodium under high pressure, Phys. Rev. Lett. 108 (2012) 115701.

[15] M.-s. Miao, R. Hoffman, High-Pressure Electrides: The Chemical Nature of Interstitial Quasiatoms, J. Am. Chem. Soc. 137 (2015) 3631-3637.

[16] Y. Li, Y. Wang, C. J. Pickard, R. J. Needs, Y. Wang, Y. Ma, Metallic Icosahedron Phase of Sodium at Terapascal Pressures, Phys. Rev. Lett. 114 (2015) 125501.

[17] I. I. Naumov, R. J. Hemley, Origin of Transitions between Metallic and Insulating States in Simple Metals, Phys. Rev. Lett. 114 (2015) 156403.

[18] http://www.crystallography.net/cod/9008545.html.

[19] B. Militzer, Ph.D. Thesis, University of Illinois at Urbana-Champaign (2000).

[20] B. Militzer, Computation of the high temperature Coulomb density matrix in periodic boundary conditions, Comput. Phys. Commun. 204 (2016) 88-96.

[21] D. M. Ceperley, Fermion nodes, J. Stat. Phys. 63 (1991) 1237-1267.

[22] http://www.msg.ameslab.gov/gamess/.

[23] J. P. Perdew, A. Zunger, Self-interaction correction to density-functional approximations for manyelectron systems, Phys. Rev. B 23 (1981) 5048.

[24] D. M. Ceperley, B. J. Alder, Ground state of the electron gas by a stochastic method, Phys. Rev. Lett. 45 (1980) 566-569.

[25] P. E. Blöchl, O. Jepsen, O. K. Andersen, Improved tetrahedron method for brillouin-zone integrations, Phys. Rev. B 49 (1994) 16223-16233.

[26] G. Kresse, J. Furthmüller, Efficient iterative schemes for ab initio total-energy calculations using a plane-wave basis set, Phys. Rev. B 54 (1996) 11169.

[27] http://opium.sourceforge.net. 